\title{
THE RATE OF GROWTH OF THE DENOMINATORS IN THE OPPENHEIM SERIES ${ }^{1}$
}

\author{
JANOS GALAMBOS
}

\begin{abstract}
A Borel-Cantelli lemma is proved for a sequence of functions of the denominators in the Oppenheim expansion of real numbers. This is then applied to the study of the rate of growth of the denominators in the above series. The laws obtained are almost sure type, that is, valid for (Lebesgue) almost all $x$ in the unit interval. The results are new even for the classical expansions of Engel, Sylvester and Cantor (product).
\end{abstract}

1. Introduction. Let $q_{n}(k)$ be a sequence of positive functions of natural numbers $k$ which take rational numbers. We call the representation

$$
x=\frac{1}{d_{1}}+q_{1}\left(d_{1}\right) \frac{1}{d_{2}}+q_{1}\left(d_{1}\right) q_{2}\left(d_{2}\right) \frac{1}{d_{3}}+\cdots
$$

of real numbers $0<x \leqslant 1$ the Oppenheim series of $x$ if the integers $d_{n}=d_{n}(x)$ are determined by the following algorithm:

$$
x=x_{1}, \quad 1 / d_{n}<x_{n} \leqslant 1 /\left(d_{n}-1\right), \quad x_{n}=1 / d_{n}+q_{n}\left(d_{n}\right) x_{n+1} .
$$

We put

$$
h_{n}(j)=q_{n}(j) j(j-1) .
$$

Throughout this paper we assume that $h_{n}(j)$ is integer valued. Under this assumption, as is easily seen, (1) and (2) are equivalent to (1) and the property below:

$$
d_{1} \geqslant 2, \quad d_{n+1} \geqslant h_{n}\left(d_{n}\right)+1, \quad n \geqslant 1 .
$$

(4) itself expresses a rate of growth for the denominators $d_{n}$. A deeper law was recently obtained in Galambos [5], where the following result is proved. Let $h_{0}(t)=1$ and, for $n=1,2, \ldots$,

$$
D_{n}=D_{n}(x)=\max \left\{R_{j}(x): 1 \leqslant j \leqslant n\right\},
$$

where

Received by the editors October 14, 1975.

AMS (MOS) subject classifications (1970). Primary 10K10.

Key words and phrases. Oppenheim series, denominators, Borel-Cantelli lemma, asymptotic laws, lim sup, lim inf, Engel series, Sylvester series, Cantor product.

1 This research was done while the author was on Research and Study Leave from Temple University and, as a Fellow of the Humboldt Foundation, he was at the Goethe University, Frankfurt am Main. 


$$
R_{j}=R_{j}(x)=d_{j} / h_{j-1}\left(d_{j-1}\right)
$$

Then, as $n \rightarrow+\infty$,

$$
\lim P\left(D_{n}<n z\right)=\exp (-1 / z), \quad z>0 .
$$

Here, and in what follows, $P()$ stands for Lebesgue measure. While (4) expresses only that, for each $j, R_{j}>1,(7)$ indicates that $R_{j}$ is "frequently very large". The aim of this note is to make the preceding statement precise by investigating the almost sure behavior of $\lim \sup R_{j}, \lim \sup \log R_{j}$ and $\limsup \log D_{n}$. The exact results are formulated in the next section.

For examples for Oppenheim series, including the classical ones, the reader is referred to Oppenheim [6] and Galambos [2]. For metric results, see Galambos [2] and [5], Schweiger [8] and Vervaat [10] (the latter two deal with the homogeneous case only, that is, where $q_{n}(k)$, and thus $h_{n}(k)$, does not depend on $n$ ). Also relevant material, in a somewhat abstract setting, is given in Galambos [3] and Schweiger [9].

2. The results. We list our results in the following theorems and corollaries.

THEOREM 1. Let $r(n)$ be a sequence of real numbers with $r(n)>1$. Then, for almost all $x, R_{n}>r(n)$ for infinitely many values of $n$ if, and only if,

$$
\sum 1 / r(n)=+\infty
$$

More precisely, if (8) holds then $R_{n}>r(n)$ infinitely often. On the other hand, if (8) fails then $R_{n}>r(n)$ can hold only for finitely many values of $n$.

COROLlaRY 1. Let $r(n)$ be an increasing sequence of real numbers with $r(1) \geqslant 1$. Then, for almost all $x, D_{n}>r(n)$ for infinitely many values of $n$ if, and only if, (8) holds.

COROllary 2. Let $r(n)$ be as in Corollary 1. Then, for almost all $x$, as $n \rightarrow+\infty$,

$$
\begin{aligned}
& \lim \sup D_{n} / r(n)=0 \quad \text { if } \sum 1 / r(n)<+\infty, \\
& \limsup D_{n} / r(n)=+\infty \quad \text { if } \sum 1 / r(n)=+\infty
\end{aligned}
$$

COROllary 3. For almost all $x$, as $n \rightarrow+\infty$,

$$
\begin{aligned}
& \limsup \frac{\log R_{n}-\log n}{\log \log n}=1 ; \\
& \limsup \frac{\log D_{n}-\log n}{\log \log n}=1 .
\end{aligned}
$$

While Corollary 3 indicates similarities of $R_{n}$ and $D_{n}$, the following theorem shows that $D_{n}$ is "smoother" than $R_{n}$.

THEOREM 2. For almost all $x$, as $n \rightarrow+\infty$,

$$
\liminf \frac{\log R_{n}-\log n}{\log \log n}=-\infty ;
$$




$$
\liminf \frac{\log D_{n}-\log n}{\log \log n}=0
$$

Corollary 3 and Theorem 2 immediately yield

Corollary 4. For almost all $x$, as $n \rightarrow+\infty, \lim \left(\log D_{n} / \log n\right)=1$.

We conclude this section with a remark. The results presented here show a remarkable similarity between the behaviour of the sequence $R_{n}$ and of the coefficients in regular continued fractions (see Galambos [4] and Philipp [7]). It would be very interesting to find a way to deduce directly the corresponding results from each other. That is, to relate somehow an arbitrary Oppenheim expansion (or even just the Lüroth expansion) to regular continued fractions through the sequence $R_{n}$.

3. Proofs. We shall need the following result of Galambos [5].

Lemma. Define the positive integer $T_{j}=T_{j}(x)$ by $T_{j}<R_{j} \leqslant T_{j}+1, j \geqslant 1$. Then $T_{1}, T_{2}, \ldots$ are stochastically independent and, for $s=1,2, \ldots$,

$$
P\left(T_{j}=s\right)=1 / s(s+1) .
$$

Proof of Theorem 1. By the Lemma,

$$
P\left(T_{n}>r(n)\right)=1 /([r(n)]+1),
$$

where $[y]$ signifies the integer part of $y$. Since $r(n) \geqslant 1$,

$$
1 /([r(n)]+1) \geqslant 1 / 2 r(n),
$$

and thus the validity of (8) implies that

$$
\sum_{n=1}^{+\infty} P\left(T_{n}>r(n)\right)=+\infty
$$

Another appeal to the Lemma and the classical Borel-Cantelli lemma yield that, for almost all $x$, infinitely of ten $T_{n}>r(n)$. But since $R_{n}>T_{n}$, we have proved that (8) implies for almost all $x$ that for infinitely many values of $n, R_{n}>r(n)$.

Conversely, since

$$
P\left(R_{n}>r(n)\right) \leqslant P\left(T_{n}>r(n)-1\right) \leqslant\{r(n)+1\}^{-1},
$$

the convergence of $\sum 1 / r(n)$ implies that

$$
\sum P\left(R_{n}>r(n)\right)<+\infty \text {. }
$$

It is well known in elementary probability theory that (9) implies that, for almost all $x, R_{n}>r(n)$ can occur only finitely many times. Theorem 1 is thus established.

Proof of Corollary 1. Let first $r(n)$ be bounded. Then (8) holds and thus Theorem 1 implies that, for almost all $x, D_{n} \geqslant R_{n}>r(n)$ for infinitely many values of $n$. 
Let now $r(n) \rightarrow+\infty$ with $n$. We shall prove that $R_{n}>r(n)$ occurs infinitely of ten if, and only if, $D_{n}>r(n)$ holds for infinitely many values of $n$. Indeed, if $R_{n}>r(n)$ then evidently $D_{n}>r(n)$. Conversely, if $D_{n}>r(n)$ then there is an $m \leqslant n$ such that $R_{m}>r(n)$. Since $r(n)$ is assumed to be increasing, $r(n) \geqslant r(m)$ and thus $R_{m}>r(m)$. Now if $r(n) \rightarrow+\infty$, then for any $n$, only finitely many times can the same $m$ be chosen with $R_{m}>r(n)$. Therefore, if infinitely many times $D_{n}>r(n)$, then there are infinitely many $m$ with $R_{m}>r(n) \geqslant r(m)$. This completes the proof of Corollary 1 .

Proof OF Corollary 2. Let $M$ be an arbitrary positive integer. If $\sum 1 / r(n)<+\infty$, we apply Corollary 1 with $r(n) / M$ for $r(n)$. We get that, as $n \rightarrow+\infty$,

$$
\lim \sup D_{n} / r(n) \leqslant 1 / M
$$

Since $M$ is arbitrary, the left-hand side above is zero. On the other hand, if $\sum 1 / r(n)$ diverges, we apply Corollary 1 with $\operatorname{Mr}(n)$ for $r(n)$. The result is that, for any $M$, as $n \rightarrow+\infty$,

$$
\limsup D_{n} / r(n) \geqslant M \text {. }
$$

This is possible only if the left-hand side is $+\infty$. The proof is completed.

Proof of Corollary 3. This is immediate by applying Theorem 1 and Corollary 1 , respectively, with $r(n)=n(\log n)^{t}$.

Proof of Theorem 2. Part (i) actually is a very weak form of the real situation. It is stated in this form only for comparison with $D_{n}$. As a matter of fact, the Lemma implies that, for almost all $x, T_{n}=1$ infinitely often, that is, $R_{n} \leqslant 2$ for infinitely many values of $n$. Hence, without any normalization, as $n \rightarrow+\infty$,

$$
\lim \inf \log R_{n} \leqslant \log 2 .
$$

For proving part (ii), we put $U_{n}=\max \left(T_{1}, T_{2}, \ldots, T_{n}\right)$. By definition,

$$
U_{n}<D_{n} \leqslant U_{n}+1 \text {. }
$$

However, one can easily deduce that a statement similar to (ii) is valid for $U_{n}$ (see Barndorff-Nielsen [1]) by the independence of the $T$ 's. Such a result, together with (10), yields (ii) and thus the proof is complete.

As remarked earlier, Corollary 4 is immediate from Corollary 3 and Theorem 2.

\section{REFERENCES}

1. O. Barndorff-Nielsen, On the rate of growth of the partial maxima of a sequence of independent identically distributed random variables, Math. Scand. 9 (1961), 383-394. MR 25 \#2625.

2. J. Galambos, The ergodic properties of the denominators in the Oppenheim expansion of real numbers into infinite series of rationals, Quart. J. Math. Oxford Ser. (2) 21 (1970), 177-191. MR 41 \#3423.

3. - On infinite series representations of real numbers, Compositio Math. 27 (1973), 197-204. MR 48 \#11026.

4. - An iterated logarithm type theorem for the largest coefficient in continued fractions, Acta Arith. 25 (1973/74), 359-364. MR 49 \#8952. 
5. - Further ergodic results on the Oppenheim series, Quart. J. Math. Oxford Ser. (2) 25 (1974), 135-141. MR 50 \#260.

6. A. Oppenheim, The representation of real numbers by infinite series of rationals, Acta Arith. 21 (1972), 391-398. MR 46 \#8982.

7. W. Phillip, A conjecture of Erdös on continued fractions, Acta Arith. 28 (1975/76), 379-386.

8. F. Schweiger, Metrische Sätze über Oppenheimentwicklungen, J. Reine Angew. Math. 254 (1972), 152-159. MR 45 \#6781.

9. - Gedämpfte zahlentheoretische Transformationen, Monatsh. Math. 79 (1975), 67-73.

10. W. Vervaat, Success epochs in Bernoulli trials (with applications in number theory), Math. Centre Tracts, no. 42, Math. Centrum, Amsterdam, 1972. MR 48 \# 7331.

Department of Mathematics, Temple University, Philadelphia, Pennsylvania 19122 